\title{
EL SISTEMA ET L'ATTACHEMENT À LA DÉFAMILIARISATION: ENSEIGNEMENT ET APPRENTISSAGE DE LA MUSIQUE SYMPHONIQUE A SANTA ROSA DE AGUA, VENEZUELA
}

\author{
EL SISTEMA E A VINCULAÇÃO À DESFAMILIARIZAÇÃO: ENSINO E APRENDIZAGEM DE MÚSICA \\ SINFÓNICA EM SANTA ROSA DE AGUA, VENEZUELA \\ EL SISTEMA AND ATTACHMENT TO DEFAMILIARIZATION: TEACHING AND LEARNING OF \\ SYMPHONIC MUSIC IN SANTA ROSA DE AGUA, VENEZUELA
}

EL SISTEMA Y APEGO A LA DESFAMILIARIZACIÓN: ENSEÑANZA Y APRENDIZAJE DE MÚSICA SINFÓNICA EN SANTA ROSA DE AGUA, VENEZUELA

\author{
Alix Sarrouy \\ Universidade Nova de Lisboa, Centro Interdisciplinar de Ciências Sociais, Portugal
}

\begin{abstract}
RÉSUMÉ: Partant d'une recherche ethnographique faite au Venezuela en 2015 dans une école d'El Sistema, programme d'enseignement de la musique symphonique, cet article est une analyse pragmatiste (Dewey, 2010) de ce qu'est l'enseignement et l'apprentissage musical dans un tel milieu. Les résultats rendent compte d'interactions sociales collectives mais concrètes entre élèves et professeurs, spécifiques au contexte symphonique. L'analyse des données s'appuie sur le concept de «attachement» (Hennion, 2004) à ce qu'il y a de plus familier et de rassurant, puis sur le concept de "défamiliarisation» (Shklovsky, 2008) face à ce qu'il y a de plus étranger et de non-familier. Paradoxalement, sur le terrain du concret, ces deux conceptualisations sont autant interdépendantes qu'en tension constante. Cela crée des contrastes émotifs vécus au quotidien, pouvant renforcer la cohésion sociale et la continuation des écoles de musique.
\end{abstract}

Mots-clés: El Sistema, éducation musicale, action collective, attachements, défamiliarisation.

RESUMO: Com base numa investigação de caracter etnográfico realizada em 2015 no El Sistema, programa venezuelano de ensino de música sinfônica, este artigo é uma análise pragmatista (Dewey, 2010) do que é ensinar e aprender música nesse contexto. Os resultados revelam interações sociais concretas entre alunos e professores, específicas ao contexto sinfónico. A análise dos dados apoia-se no conceito de "vinculação" (Hennion, 2004) ao que há de familiar e de reconfortante, e no conceito de "desfamiliarização" (Shklovsky, 2008) face ao que há de estranho e de não familiar. Paradoxalmente, no campo do concreto, estas duas conceptualizações estão simultaneamente interdependentes e em tensão constante. Vivem-se contrastes quotidianamente, podendo fortalecer a coesão social e a continuação das escolas de música.

Palavras-chave: El Sistema, educação musical, ação coletiva, vinculação, desfamiliarização.

ABSTRACT: Based on an ethnographic investigation carried out in 2015 within El Sistema, a Venezuelan program for teaching symphonic music, this article is a pragmatic analysis (Dewey, 2010) of what it is to teach and learn music in this context. The results reveal concrete social interactions between students and teachers, specific to the symphonic context. Data analysis is based on the concept of 'attachment' (Hennion, 2004) to what is familiar and comforting, and the concept of 'defamiliarization' (Shklovsky, 2008) in view of what is strange and unfamiliar. Paradoxically, these two conceptualizations are simultaneously interdependent and in constant tension. Contrasts are experienced every day, which can strengthen social cohesion and the continuation of music schools.

Keywords: El Sistema, musical education, collective action, bonding, defamiliarization.

RESUMEN: Basado en una investigación etnográfica realizada en 2015 en El Sistema, un programa venezolano para la enseñanza de música sinfónica, este artículo es un análisis pragmático (Dewey, 2010) de lo que es enseñar y aprender música en este contexto. Los resultados revelan interacciones sociales concretas entre estudiantes y profesores, específicas del contexto sinfónico. El análisis de datos se basa en el concepto de "apego" (Hennion, 2004) a lo familiar y reconfortante, y el concepto de "desfamiliarización" (Shklovsky, 2008) en vista de lo extraño y desconocido. Paradójicamente, en el campo del concreto, estas dos conceptualizaciones son simultáneamente interdependientes y en constante tensión. Diariamente se viven contrastes que pueden fortalecer la cohesión social y la continuidad de las escuelas de música.

Palabras-clave: El Sistema, educación musical, acción colectiva, vinculación, desfamiliarización. 


\section{Introduction: El Sistema et la musique comme outil}

Le point de départ de cet article est une recherche ethnographique faite dans le cadre de mon doctorat en sociologie de l'art et de la culture (2013-2017). À travers une recherche qualitative et comparative, mon projet consistait à essayer d'approfondir les connaissances sur l'usage de la musique comme outil d'enseignement et d'émancipation des populations socio-économiquement défavorisées dans trois pays: Venezuela, Brésil, Portugal (Sarrouy, 2016, 2017a). ${ }^{6}$ En 2015 j'ai passé trois mois au Venezuela pour y faire une recherche et mieux comprendre les types et les rôles des interactions sociales au sein de ce programme d'éducation musicale qui avait acquis une réputation mondiale. El Sistema est le nom usuel donné au programme d'El Sistema Nacional de Orquestas y Coros Juveniles e Infantiles de Venezuela, géré par la Fondation Musicale Simón Bolívar (FundaMusical Bolívar), depuis 2012 sous la tutelle du Ministerio del Poder Popular del Despacho de la Presidencia y Seguimiento de la Gestión de Gobierno (Rojas, 2010). II s'agit d'un programme d'enseignement musical et de création d'orchestres symphoniques pour des jeunes essentiellement issus de milieux en situation socio-économique défavorisée. Créé par Maestro José Antonio Abreu († 2018), économiste et musicien, ce programme est devenu un exemple dans le monde car il a réussi à se développer exponentiellement depuis 1975. El Sistema rassemble plus de quatre-cents «noyaux», traduction directe du mot núcleos en espagnol, c'est à dire des lieux où la musique est enseignée. Ils sont répartis par écoles, par villes et par régions. Les élèves peuvent être inscris dès l'âge de trois ans. Plus ils avancent et montent de niveau d'orchestre, plus le travail est intense : jusqu'à cinq heures de musique par jour, six jours par semaine (Agrech, 2018; Creech, 2016; Sarrouy, 2017a, 2017b; Uy, 2012).

La mission principale d'El Sistema est d'occuper de façon pédagogique la jeunesse, particulièrement celle en situation défavorisée, c'est-à-dire des jeunes issus de familles à faibles ressources socio-économiques et, pour cette raison, exposés à des déviances sociales comme la violence, le trafic de drogue ou la prostitution dans les barrios pauvres des grandes agglomérations. La musique, par la pratique instrumentale et vocale, sert d'outil pour révéler et développer le potentiel de chacun individuellement et en collectif. Elle permet d'avoir un nouveau point d'accroche et des projets futurs. Ce sont là les résultats les plus visibles mais il y a d'autres impacts sur les nouvelles générations, tout aussi positifs mais plus discrets, car ce travail touche un ensemble de facteurs humains et sociétaux que j'aurai l'occasion de développer au cours de cet article. Tocar, cantar y luchar veut dire Jouer, chanter et lutter. C'est la devise d'El Sistema, car non seulement les élèves apprennent à jouer de la musique, mais ils apprennent aussi à briser les reproductions sociales menant à des déviances. El Sistema dit rechercher bien plus que des résultats de qualité musicale, le souhait est d'atteindre des objectifs sociaux d'éthique et de citoyenneté à travers la pratique collective de la musique.

À travers sa communication, notamment son site Internet ${ }^{7}$, El Sistema explique que savoir écouter est un apprentissage fondamental pour un futur interculturel entre personnes et entre peuples. La bonne écoute de l'autre permettrait le dialogue, la compréhension d'un message transmis, et donc le vrai partage d'informations. Le travail en orchestre enseigne les règles basiques d'un dialogue, c'est-à-dire: l'écoute; la prise de parole constructive comme complément

\footnotetext{
${ }^{6}$ Thèse de doctorat en cotutelle: Université Sorbonne Nouvelle - Paris 3 (France) et Université de Minho (Portugal). Codirection par Antoine Hennion (CSI, CERLIS) et Beatriz Padilla (ICS, CICS.NOVA).

${ }^{7}$ Site officiel: www.fundamusical.org.ve
} 
de l'autre; la prise de conscience des droits et des devoirs au sein d'un collectif ou d'une société. Pour que l'orchestre fonctionne et pour une bonne interprétation musicale, il doit y avoir comme principes de base mais à atteindre en permanence: l'égalité; la complémentarité entre tous les éléments; l'écoute de soi et de l'autre; un(e) chef(fe) d'orchestre qui dirige les musiciens musicalement et humainement (Govias, 2011) ${ }^{8}$.

El Sistema se veut être une fenêtre qui permet à la jeunesse vénézuélienne d'avoir des objectifs et de les atteindre. On y enseigne supposément l'excellence musicale tout en transmettant les valeurs du travail, de la persévérance, du respect de soi et de l'autre afin de lier l'expérience artistique à l'évolution sociale. Des chefs d'orchestre de niveau mondial ont reconnu l'importance et la qualité du travail fait au Venezuela. C'est le cas de Maestro Simon Rattle qui, à propos d'El Sistema, a dit que "c'est mondialement le plus important travail fait autour de la musique», et de Maestro Claudio Abbado qui définissait El Sistema comme «le meilleur exemple à suivre par tous les pays» (Arvelo, 2006). Ces phrases et témoignages de grandes figures du monde dit «classique», aussi sincères soient-ils, font le régal des médias et servent d'outils de légitimation et de communication d'El Sistema.

Cependant, en 2014, à l'apogée de la reconnaissance mondiale d'El Sistema, une voix critique venant du monde académique, le chercheur anglais Geoff Baker, publie un livre accusant et condamnant de possibles facettes cachées du projet vénézuélien (2014). Les répercussions sont directes et de fortes polarisations «pour vs contre» se font sentir. Baker est notamment rejoint par un professeur américain Lawrence Scripp, du New England Conservatory, qui a réalisé une interview d'un ancien élève d'El Sistema, actuellement violoniste à l'Orchestre du Ballet de Pennsylvanie, soutenant les accusations (2015). D'autre part, des figures internationales ont soutenu El Sistema. En effet, El Sistema a bénéficié de représentants très fidèles et de longue date, avec comme principaux communicants les auteurs américains Tricia Tunstall et Eric Booth (2016), ainsi que l'anglais Marshall Marcus, ancien Directeur Musical du Southbank Center à Londres. Ce sont des acteurs très présents dans le «mouvement mondial d’El Sistema», notamment par la médiation de livres, d'articles et des conférences sur les idées, les méthodes et acteurs du programme vénézuélien.

Il y a donc plusieurs avis et courants autour d'El Sistema. Les opinions sont contrastées, passant de la critique superlative à l'hagiographie dévote. Ni l'un ni l'autre de ces extrêmes ne m'ont intéressé comme point de départ, d'ailleurs ma recherche était déjà bien engagé avant 2014. De même, le présent article ne se penche pas sur le contexte politique instable et précaire de ces dernières années au Venezuela. J'en suis bien conscient et cela mérite un autre article spécifique, notamment parce-que les changements de cette dernière décennie ont beaucoup affecté la population vénézuélienne (plus de cinq millions d'émigrés) avec de graves impacts sur la structure humaine et logistique d'El Sistema. Sans oublier le contexte dramatique que traverse actuellement le Venezuela, je propose d'en revenir à la musique et aux faits sociaux que j'ai eu la possibilité d'observer et de vivre en 2015 dans le quartier de Santa Rosa de Agua. J'appuie cet article sur ce terrain de recherche ethnographique au Venezuela, mais j'inclus quelques exemples vécus chez d'autres programmes qui s'en inspirent, au Brésil, en France et au Portugal.

${ }^{8}$ Voir aussi le site officiel de la plateforme Social Impact of Making Music (SIMM) www.simm-platform.eu 
Cet article est divisé en quatre parties qui ont pour fil conducteur l'orchestre symphonique comme instrument pédagogique et comme contexte d'expérimentations sociales. Je propose de commencer par (1) une analyse des aspects concrets rendus possibles à travers l'orchestre symphonique en tant qu'outil d'enseignement et d'apprentissage. Ensuite (2), je m'intéresse à l'action collective en donnant quelques exemples de situations précises rendues possibles par le format orchestral, ainsi que des exemples d'aptitudes individuelles et collectives auxquelles sont soumis l'ensemble des acteurs. À la troisième étape (3) cherche rendre visible ce qui permet que les acteurs d'un orchestres "fassent corps ${ }^{9}$, en insistant sur le rapport entre l'individu et le collectif dans ce format de mini société musicale. La quatrième partie (4) est une proposition d'ouverture de l'analyse en passant par la notion de «défamiliarisation » opérée par le formaliste russe Shklovsky dans L'art comme procédé (2008). L'article se termine par une partie intitulée Discussion et Conclusion, où je propose de partir des données récoltées sur le terrain pour approfondir le rapport paradoxal entre les notions de «défamiliarisation» et de "attachement» (Hennion, 2015). Leur complémentarité ainsi que les tensions continues me semblent être au cœur de ce qui renforce le liens entre l'ensemble des acteurs intervenant dans un núcleo et dans un orchestre symphonique.

\section{Les possibles de l'orchestre symphonique}

L'exemple d'El Sistema est reproduit dans plus de soixante pays ${ }^{10}$, notamment en France par les projets Démos, Sistema France, Tutti Passeurs d'Art; et au Portugal avec Orquestra Geração et GeraJazz (Amico et al., 2018; Creech, 2016; Mota \& Teixeira Lopes, 2017; Sarrouy, 2011; Silberman, 2013). El Sistema propose de se servir de la musique symphonique pour prévenir et traiter des problèmes d'ordre social, économique et culturel dès le plus jeune âge. Cela mène à poser la question de l'emploi de la musique et plus généralement de l'art comme outil de travail auprès des populations dans un territoire spécifique. L'art peut-il être un outil face à des problèmes socioculturels? De quelle façon? De quels outils parlons-nous : l'objet d'art in fine ou le processus artistique dans son ensemble? Quelles sont les spécificités du travail musical en orchestre symphonique? Le travail en orchestre est-il plus qu'esthétique? De quelle façon procède-t-il concrètement?

Je propose de partir d'une analyse pragmatiste (Dewey, 2010; Shusterman, 1991) de ce qu'est un orchestre symphonique: l'observation ethnographique au cours de plusieurs mois auprès d'orchestres symphoniques m'a permis de relever certaines des caractéristiques intrinsèques qui permettent au format orchestral puis à la musique symphonique d'être des outils effectifs dans le travail socioculturel d'El Sistema. Les points qui suivent seront précis et courts, par petits paragraphes, résultant de mes observations ethnographiques. Ils serviront à mettre en évidence des aspects très concrets de l'enseignement musical mais qui sont souvent oubliés lorsqu'il nous faut rendre compte de ce qui se passe dans une salle de classe (Hennion, 1988).

L'orchestre symphonique est avant tout constitué d'un ensemble de musiciens qui obéissent à certaines règles générales liées: au positionnement des interprètes (selon le niveau musical et le

\footnotetext{
${ }^{9}$ «Faire corps avec»: ne faire qu'un avec quelqu'un d'autre; adhérer à quelque chose. www.larousse.fr/dictionnaires/francais/corps/

10 Voir le site officiel de Sistema Global : www.sistemaglobal.org / Voir aussi le site officiel des newsletters The Ensemble, à propos des programmes inspirés par El Sistema dans le monde : www.theensemblenewsletters.com
} 
lien hiérarchique au Maestro); à l'emplacement des instruments (selon les familles, leur fonction et l'intensité du son qu'ils produisent); à la très grande variété d'instruments musicaux qui permettent d'interpréter un large éventail de compositions dirigées par un chef d'orchestre. Le format orchestral permet de regrouper entre 80 et 120 musiciens autour d'une même composition et d'un même chef d'orchestre. Pour certaines occasions, El Sistema regroupe 200 musiciens dans un même orchestre. Ce n'est pas le cas de beaucoup d'autres genres musicaux.

Tout comme ce qui se passe dans d'autres rassemblements humains, l'orchestre permet de créer et de partager des affinités, comme par exemple la passion pour un instrument ou un compositeur. Dans un orchestre, il y a des dizaines de petits groupes sociaux possibles, ce qui crée du lien social. L'orchestre permet de travailler en collectif et donc de rechercher une motivation dans le groupe. C'est bien différent du travail individuel et même des petits ensembles. L'orchestre dans sa totalité transmet beaucoup plus de force que la somme de ses parties, et ceci même le plus jeune musicien le ressent. Le travail collectif rendu possible en orchestre est très important pour des jeunes élèves recherchant des motivations et des exemples à suivre au sein même du groupe. L'ensemble des relations personnelles existant dans l'orchestre peut être un moteur de dynamisation pour chacun. L'orchestre est perçu comme une mini-société multiculturelle avec une grande variété d'individus, d'instruments, d'emplacements et de fonctions possibles. La prise en compte et le respect de cette diversité renforcent aussi le lien social.

Comme en société, l'orchestre suit un système hiérarchique. C'est une forme de hiérarchie qui peut être «harmonieuse» et préétablie. Concrètement: le chef d'orchestre; le premier violon; les chefs de pupitres; puis chaque musicien de chaque famille d'instruments (cordes, bois, cuivres, percussions...). Tous dépendant les uns des autres. Savoir vivre et s'exprimer en respectant différentes formes de hiérarchie sont des valeurs fondamentales en société, possibles de transmettre aux jeunes musiciens. L'orchestre permet de travailler l'écoute. Plus il est grand, plus l'écoute doit être fine et attentive afin de trouver sa place: accordage; tonalité; hauteur et longueur du son, entre autres. Une meilleure écoute peut mener à une meilleure entente en société, c'est une autre capacité fondamentale pour les futurs adultes.

Dans un orchestre symphonique les rôles varient pendant l'interprétation d'une même composition, par exemple: chacun peut avoir un moment de solo, dans le sens où un instrument spécifique peut être mis en avant pendant quatre mesures, puis ensuite s'effacer parmi les autres. Il y a donc une conscience du mélange qui vise l'harmonie puis de la mise en avant momentanée par un solo. Cet aller-retour de compétences peut être formateur dans des sociétés où les rôles ne sont pas non plus figés. Pour le musicien, le travail en orchestre implique un effort de gestion de quatre composantes: soi-même, par le son que l'on produit; la partition; les autres musiciens, par le son qu'ils produisent; et le chef d'orchestre qui dirige. L'attention constante et le respect de ces quatre composantes obligent le musicien à un effort de concentration et de recherche constante d'harmonie.

L'orchestre symphonique permet de développer des formes de travail très variées selon le positionnement de chaque musicien. Par exemple, un chef de pupitre doit être un médiateur entre le chef d'orchestre et son pupitre. À ce propos, j'ai eu l'opportunité d'assister aux conseils donnés par un professeur à son élève chef de pupitre de dix-sept ans. Le professeur lui a fait prendre conscience de l'importance de son rôle et de la façon de le mener, en lui disant que: le chef de pupitre doit tout faire pour que son pupitre joue toujours à son meilleur niveau; il ne faut pas trop 
diriger son pupitre verbalement car ses membres n'aiment pas être conseillés et mis en défaut face aux autres; pour cela, il faut utiliser le regard et la démonstration. Il faut donc faire comprendre plutôt que d'expliquer de façon incisive; il faut être en permanence attentif au chef d'orchestre et à son pupitre. On peut en conclure que le chef de pupitre est un médiateur et que ces conseils sont transposables à d'autres formes de médiation culturelle (Caune, 2006; Hennion, 2007; Lafortune, 2012).

Les différentes positions de «pouvoir« dans l'orchestre (chef de pupitre, $1^{\text {er }}$ violon, chef d'orchestre) rendent opportuns certains débats internes afin de transmettre des valeurs de vie en société, par exemple: à propos de l'âge, lorsque le professeur choisit temporairement un violoniste de treize ans comme chef de pupitre dans un groupe où les plus âgés ont seize ans, ces derniers vont devoir accepter qu'un plus jeune les dirige et inversement le plus jeune va devoir assumer ce positionnement; à propos du genre, lorsque le chef de pupitre est une jeune fille, les garçons du groupe doivent assumer le fait qu'elle puisse et sache les diriger.

Le répertoire d'un orchestre symphonique est très varié, ce qui permet le choix de compositions selon la population que représentent les musiciens. Par exemple dans le cas des orchestres symphoniques juvéniles du Venezuela (avec des jeunes entre seize et vingt-cinq ans c'est le cas pour l'Orchestre Teresa Carreño, au Venezuela), le choix du répertoire révèle souvent une attention à la dynamique des compositions. Les Vénézuéliens penchent plus vers des compositions rythmiques et pleines d'intensité comme le Mambo de Leonard Bernstein, le Danzon no2 d’Arturo Marquez ou la Symphonie No. 4 de Tchaïkovski. Les jeunes musiciens sentent nettement plus de motivation pour ce genre de répertoire, ce qui rend la diversité de choix encore plus importante selon l'âge et le niveau musical.

Cette liste non exhaustive de ce qui est pragmatiquement rendu possible par le format d'orchestre symphonique dans un núcleo, mais pas seulement, met en évidence des dispositions intrinsèques d'ordre formel: le collectif; la diversité d'instruments et de fonctions; la complémentarité entre les éléments du collectif; la hiérarchie; parmi bien d'autres. Ces dispositions permettent un ensemble de situations à travers lesquelles se développent en parallèle, et de façon complémentaire, les aspects sociaux et artistiques chez les jeunes apprentis. L'enfant qui intègre un nouvel orchestre dans son école peut choisir un instrument dans l'éventail symphonique, il peut compter sur le travail en collectif qui évite l'isolement, compter sur le partage des problèmes d'apprentissage avec son collègue de pupitre, il peut aussi être sûr qu'il ne sera pas le seul à être mis en avant par un solo ou mis en retrait pour accompagner.

\section{Symphonie de l'action collective}

L'action collective (Becker, 1974; Cefaï, 2007) à laquelle sont invités les jeunes élèves permet tout un ensemble d'opérations à caractère pédagogique ayant un impact direct sur l'individu interprète et citoyen. Entrons dans une classe d'un núcleo d'El Sistema ${ }^{11}$, afin de comprendre plus précisément le rôle essentiel des professeurs et des élèves dans ce travail qui mêle les pédagogies musicales, sociétales et éthiques pour des objectifs socio-artistiques profondément mêlés.

\footnotetext{
${ }^{11}$ De janvier à avril 2015 j'ai fait d'intenses recherches ethnographiques sur un seul núcleo dans le quartier Santa Rosa de Agua, ville de Maracaibo, à l'ouest du Venezuela (Sarrouy, 2018). Ce choix fut en accord avec la direction d'El Sistema selon ma volonté d'étudier un núcleo d'une grande ville qui ne soit pas Caracas car la plupart des chercheurs y font leurs recherches et il y a plus de "contrôle" sur leur travail.
} 
L'accueil - C'est le premier contact entre le professeur et les jeunes élèves musiciens. II commence par des questions comme: «Bonjour, comment allez-vous?»; «Toi Julia, comment vas-tu?»; "Qu'as-tu fait ce weekend?», ces questions sont si simples, apparemment si banales et pourtant essentielles. Elles peuvent ne pas être prises au sérieux par les élèves au début, mais leur récurrence se transforme en rituel permettant de créer un premier lien en début de classe. Ces questions en début de session apportent souvent humour et décontraction avant le travail sérieux. Elles permettent aussi aux élèves de s'exprimer et au professeur de prendre la «température» du collectif qui se trouve en face de lui. C'est également un moment d'une extrême importance pour certains éléments plus vulnérables du groupe d'élèves issus d'un milieu socialement et psychologiquement instable. Cette qualité d'accueil peut avoir des effets subtils et importants pour le bien-être des enfants, et des conséquences sur leur apprentissage et leur insertion dans le groupe.

L'assise - S'asseoir sur une chaise est un acte banal, dans un enseignement musical ceci a pourtant beaucoup d'importance. Pour un violoncelliste par exemple, la chaise sert à être à un bon niveau pour jouer de son instrument, elle ne sert pas à s'adosser. Tous doivent s'asseoir au bord de leur chaise, la colonne vertébrale droite et le menton vers le haut. Ce qui peut paraître de rigueur militaire a une triple importance: ne pas se faire mal physiquement à force de jouer longtemps; avoir une qualité de son consistante; inciter à une discipline qui vise à unir l'orchestre dans un même effort qui commence par le physique puis évolue à travers le mental et le musical. Cette union d'efforts est essentielle pour la transmission de la volonté commune d'atteindre un résultat commun. La plupart des professeurs passent beaucoup de temps sur le positionnement du corps, posant dès le début des règles de rigueur et de respect. C'est très profitable pour un enfant qui n'est pas habitué à ce genre de cadrage dans son entourage familial et social. D'ailleurs, c'est très visible, pour certain l'apprentissage de l'assise calme et maitrisée prend des mois.

S'accorder - S'accorder est, comme l'assise, l'une des étapes essentielles du travail musical en groupe. Bien plus qu'une étape individualiste elle est faite en collectif, tous doivent être accordés entre eux, en se basant sur le $L a$ du hautbois quand l'orchestre est au complet. Cette première étape permet de se concentrer, de s'écouter, de s'observer, et de trouver ainsi la communion par la note. On part d'une même base qui sert de premier lien entre tous les musiciens. Ce lien se fait entre musiciens mais aussi entre individus partageant un même espace, une même assise, une même note de départ et une même musique. Dans un objectif musical, le collectif s'accorde à plusieurs niveaux. Pour l'enfant issu d'une structure familiale et d'un environnement instables, le fait de trouver un point d'accord autour du La permet d'atteindre un confort musical et physique essentiel pour le développement de son travail personnel puis collectif.

Le sens de la responsabilité - La responsabilisation des jeunes élèves musiciens est très caractéristique des programmes inspirés d'El Sistema, car les professeurs et les administrateurs ne sont pas les seuls porteurs du projet. Tous les élèves et parents le sont également, ils doivent pouvoir être fiers du projet qu'ils ont intégré, être conscients d'où cela peut les mener, et donc lutter collectivement pour atteindre leurs objectifs - Tocar, cantar y luchar comme le dit le slogan d'El Sistema. Cette responsabilisation collective est aussi une méthode de pédagogie mise en avant par les professeurs. Par exemple lors d'un cours pour les cordes dirigé par le professeur Ivana 
Dudnik $^{12}$, l'un des élèves arrive en retard alors que tous les autres sont déjà au travail. Dès que le retardataire entre dans la salle, les autres élèves s'arrêtent de jouer et se mettent à hocher la tête d'un signe de négation, puis à faire «tsi tsi tsi» pour montrer le mécontentement collectif sur ce retard. Ce n'est donc pas seulement le professeur qui est en désaccord avec les retards, c'est toute la classe qui n'admet pas ce genre de comportement en le faisant savoir. Pour le retardataire ce n'est pas que le discours habituel du professeur qu'il doit écouter, c'est le regard et la désapprobation de toute une classe composée par ses pairs qu'il doit affronter. Ainsi chacun est responsable de ce qui se passe dans le groupe.

Mélange des âges - L'âge des enfants est souvent le critère de formation de groupes de travail, par exemple des tranches de 8-12 ans ou 13-16 ans, ce qui permet un mélange d'âges, de savoirs et de mentalités. L'objectif pédagogique est d'inciter les plus grands à servir d'exemple et à enseigner au plus petits. Souvent les jeunes arrivés sont timides ou inquiets, et le fait de reconnaître le visage d'un ami un peu plus âgé réconforte. C'est un moteur d'apprentissage. Ceci permet de responsabiliser les plus grands, ce qui est très important dans le cas des plus perturbateurs souvent en attente d'attention et pour lesquels cette responsabilisation surveillée mène à de très bons résultats. Le fait de mélanger les âges permet aussi de mêler les caractères et les façons de penser. Si des frictions au sein du groupe existent, elles servent de facteur d'apprentissage de la vie en société avec ses différences et ses forces.

Confiance - La confiance en soi dans un groupe de travail est une caractéristique développée par l'ensemble des professeurs des projets étudiés, notamment par la méthode Serioso de Richard Young ${ }^{13}$. Cet altiste de renom international a créé une technique d'enseignement pour les cordes en musique de chambre. II intervient dans plusieurs projets inspirés par El Sistema en Amérique Latine. Sa méthode est basée sur un groupe de seize instrumentistes à cordes, divisible par quatre. Chaque sous-groupe est composé de quatre musiciens - deux violons, un alto et un violoncelle par exemple. Les seize musiciens sont toujours regroupés mais il n'y a qu'un seul quatuor qui joue à la fois, pendant que les autres assistent. Le travail de musique de chambre développé par Richard Young implique que chaque musicien soit confiant en son jeu car il ne peut le cacher au milieu de trente autres instruments à cordes, comme en orchestre. En quatuor, le musicien est plus exposé, il faut donc développer confiance et assurance pour chaque note jouée. Pendant qu'un quatuor joue, les douze autres musiciens doivent écouter les quatre interprètes pour comprendre leurs qualités et leurs défauts. L'objectif est de développer le sens de l'écoute et le sens critique. Les avis peuvent être partagés à chaque pause. Les quatuors tournent constamment, obligeant chacun à jouer et s'écouter, ainsi les informations circulent au sein du groupe de seize, ce qui accroît l'esprit de cohésion. Les résultats sont rapidement perceptibles lors du concert de présentation au bout de deux semaines de travail.

II ressort de ces actions à caractère pédagogique que c'est à travers l'ensemble du processus artistique que se fait le travail de développement social et culturel auprès des apprentis musiciens,

\footnotetext{
12 Ivana Dudnik était professeure de violoncelle et d'orchestre de cordes au Neojiba. Pendant l'été 2010 j'ai fait trois mois de recherches ethnographiques dans ce programme bahianais dirigé par Maestro Ricardo Castro et très inspiré d'El Sistema. J'ai ensuite fait une deuxième recherche ethnographique à Salvador de Bahia, cette fois dans le núcleo du quartier périphérique Bairro da Paz en 2015. Site officiel du projet brésilien: www.neojiba.org

${ }^{13}$ Richard Young est un altiste américain de renom international (Vermeer Quartet). En parallèle il développe un travail auprès de populations socio économiquement défavorisées, notamment à Chicago à la People's Music School. Plus récemment, il a participé à plusieurs projets inspirés d'El Sistema, comme YOURS Project à Chicago, Neojiba à Salvador de Bahia et BATUTA en Colombie.
} 
tant au niveau individuel que collectif. Ce n'est pas seulement l'œuvre finale présentée en concert qui compte dans l'émancipation des jeunes musiciens, c'est tout le processus de création artistique à développer dans le long terme. Sont alors fondamentaux les détails infinis d'apprentissage en collectif rendu possible grâce à la forme pragmatique de l'orchestre symphonique et au talent pédagogique de tous les intervenants dans ce processus. Avant qu'il y ait un enseignement sur le fond et la complexité de l'esthétique musicale, il ressort de ces actions que les professeurs accordent une attention particulière aux étapes qui rendent l'élève confiant, motivé et intéressé, pour qu'il se sente membre intégrant d'un ensemble qui avance vers un même objectif qui dépend de chacun.

\section{L'art de faire corps en orchestre}

Le processus artistique commence dès le premier jour où les enfants intègrent un orchestre. Ainsi, El Sistema et les programmes qui s'en inspirent valorisent toutes les étapes. II n'y a pas de temps perdu puisque c'est l'ensemble de ce qui forme l'être qui va permettre d'aller plus loin dans l'interprétation musicale individuelle (e.g. responsabilisation et confiance) et collective (e.g. être accordé et écouter l'autre). Avant d'atteindre le niveau musical des meilleurs orchestres d'El Sistema, comme le Simon Bolivar et le Teresa Carreño, il y a une attention profonde envers la forme et la structure de l'orchestre en tant qu'outil malléable et adaptable pour la création de situations pédagogiques complémentaires aux niveaux social et artistique, tous deux esthétiques par ailleurs.

Maestro Abreu, fondateur d'El Sistema, percevait l'orchestre comme une «mini société» (Smaczny \& Stodmeier, 2009). Toute une série de face à face y sont provoqués, qu'ils soient d'ordre social (l'exemple du mélange des âges et des genres dans les pupitres, mentionné précédemment) ou d'ordre technique (une composition exigeante pour le doigté ou pour le souffle), mais aussi purement esthétique (une composition française de la période Romantique alors que l'élève a 14 ans et qu'il a grandi à Caracas au XXIe siècle). L'orchestre est un modèle réduit de société avec toute une série de «chocs» d'ordres sociétal et esthétique (Eyries, 2014) ${ }^{14}$. Ces chocs sont comme des vaccins: en petite dose ils provoquent un court malaise puis servent de prévention aux "affections» et "contagions». Le maniement de ces «chocs» révèle le pouvoir qu'ont les formateurs sur les jeunes musiciens, mais qui décide de ce qui est "affection» et de ce qui est «contagieux»? La réponse est profondément culturelle au sens anthropologique, propre à chaque territoire et c'est pour cela qu'El Sistema est difficile à reproduire dans d'autres pays sans une adaptation des méthodes - l'attention à la médiation y est donc essentielle (Sarrouy, 2017b).

El Sistema fait en sorte que chaque orchestre fasse corps et qu'il dispose de sa propre technique (Mauss, 1950) selon le territoire, la population, les objectifs. Une reproduction prosaïque et non attentive aux médiations pragmatiques analysées plus haut n'est que tentative d'imitation de la forme oubliant le fond. C'est copier une structure formelle, un squelette, en laissant de côté les médiations subtiles qui lui donnent chair. Ce squelette, cette structure formelle basique mise en évidence plus haut, permet un ensemble de chocs qui, grâce aux médiations,

${ }^{14}$ Référence aux «choc esthétique», concept clé pour la pensée et l'action d'André Malraux à l'origine du Ministère de la Culture en France, en 1958, et des Maisons de la Culture. Il peut aussi être intéressant de prolonger la réflexion sur la notion de «choc» grâce aux écrits de Walter Benjamin, notamment le texte "Sur quelques thèmes baudelairiens» (2000). 
gagne petit à petit une «chair sociale» et artistique lui permettant de se mouvoir dans les champs sociaux et musicaux. Ce mouvement est simultanément personnel et collectif.

\section{Ouverture: le rôle de la défamiliarisation dans l'attachement}

L'analyse pragmatiste du núcleo Santa Rosa de Agua, permet de mettre en évidence la perspective suivante: c'est l'ensemble du processus artistique - l'apprentissage quotidien en orchestre prenant en compte toutes les étapes -, qui sert d'outil de travail avec les jeunes élèves d'El Sistema. La forme e(s)t l'art, comme pour le sport où l'entraînement est partie intégrante de l'obtention de grands résultats. Les structures formelles proposées par El Sistema sont le processus concret et fonctionnel sur lequel vont s'installer «l'art et la manière» (Dessons, 2004), qui lie les différents éléments de cette structure à travers des médiations subtiles et fluides. Celles-ci s'appuient sur la structure pour renforcer les liens entre l'ensemble de ses parties, elles n'en sont pas séparées, elles en dépendent.

Afin d'approfondir cette analyse je propose de partir d'un article écrit par une des grandes figures du formalisme russe - Viktor Shklovsky (1893-1984). Il s'agit d'un article de 1917, qui en français s'intitule "L'art comme procédé» (2008). Ce titre met en exergue l'importance du processus dans la création artistique, tout aussi évident dans les traductions anglaises Art as Technique (Shklovsky, 1988) ou Art as device ${ }^{15}$. Le mot device contient l'idée de mécanisme, plus haut j'ai listé quelques pièces des «mécanismes» en orchestre, ainsi que leur "degré de mobilité» ${ }^{16}$.

L'article de Shklovsky est court mais riche en questionnements, j'en relève trois:

a. À propos de l'art, l'auteur ne tente pas de répondre à l'impossible "Qu'est-ce que l'art?", il nous propose plutôt d'essayer de répondre à deux questions: «À quoi sert l'art?» et «Comment sert-il à quelque chose?». C'est un transfert de problématique qui nous ramène à du concret, à une analyse pragmatique des formes/mécanismes de l'art, du formalisme des processus artistiques.

b. Cette mise en avant du processus se fait de façon concrète, cela ne signifie pas que l'on enlève ce que l'auteur appelle "caractère poétique" de chaque étape. Spécialisé en littérature, Shklovsky fait une distinction claire entre ce qui relève du langage pratique de la «prose» et ce qui relève de la «poésie». Son analyse formaliste n'est pas une défense exclusive des mécanismes, elle est une inclusion de ces mêmes mécanismes dans l'œuvre totale car eux aussi peuvent être «poétiques». L'auteur appelle à «éviter de fonctionner par application de formules», à se laisser surprendre par des possibilités «poétiques».

c. L'idée principale de son article est une mise en garde face à la banalisation des processus. Les «lois de la perception» ont tendance à devenir automatiques, pire encore, à devenir inconsciemment mécaniques, ce qui endort l'être dans sa pensée et dans son action. Afin de contredire cette tendance à la «perception automatisé», Shklovsky propose la

\footnotetext{
15 "Art as device» est une des traductions du titre russe, employée notamment dans le livre Twentieth century leterary criticism de Bijay Kumar Das (2010).

16 «Degré de mobilité» est un terme employé en théorie des mécanismes. Chaque pièce d’un mécanisme a un degré de mobilité propre, pouvant être indépendant ou conditionné par d'autres pièces d'une chaine cinématique.
} 
«défamiliarisation» rendue possible notamment par l'art lorsqu'il «obscurcit la forme, augmentant la difficulté et la durée de la perception». La «défamiliarisation» est rendue possible grâce à l'art comme «procédé contre l'usure des mots et l'automatisation».

Ces trois propositions d'analyse de la création artistique (a. À quoi sert l'art?; b. Quelle poésie dans le processus?; c. Quel est le rôle de la défamiliarisation?), permettent une analyse plus fine et concrète des orchestres symphoniques d'El Sistema en tant que mécanismes pour le développement socioculturel des jeunes musiciens.

Tout d'abord l'art de la musique symphonique sert à créer des conditions pratiques pour encadrer un grand nombre de jeunes apprentis musiciens dans un processus de création artistique. C'est ce que j'ai commencé à détailler plus haut à travers quelques exemples: la réunion de centvingt musiciens; le regroupement selon affinité, entre instruments ou entre collègues; l'expérience de vie dans un modèle réduit de société; le résultat final dépendant de chacun et de l'ensemble; parmi d'autres. La valeur poétique des pièces de la mécanique correspond au degré de mobilité de chaque personne, de chaque situation, de chaque étape. J'ai, là encore, relevé plusieurs exemples: l'accueil fait par le professeur et par les collègues; s'accorder pour rechercher une harmonie musicale puis sociale en orchestre; l'interprétation des œuvres musicales, celle-ci étant baignée de poésie lorsqu'elle n'est pas que «langage pratique» ${ }^{17}$; parmi d'autres aussi.

Pour ce qui est de la «défamiliarisation», elle semble être une constante au sein d'El Sistema: les enfants qui viennent des barrios, ainsi que leur famille et leur entourage, ne sont pas issus de milieux où la musique symphonique, le basson et le maestro, sont familiers. ${ }^{18} \mathrm{C}^{\prime}$ est également une étrangisation ${ }^{19}$ au niveau de la pédagogie faite en collectif, des compositions à jouer, des résultats pratiques à court terme, où peut régner une ambiance d'inclusion, de respect et de sécurité, aspects non ressentis dans la plupart des barrios vénézuéliens où il y a des núcleos. La défamiliarisation permet aux jeunes élèves mais aussi aux professeurs d'être constamment dans la surprise. Elle confond par le «choc» (du cadre, de l'esthétique). Elle fait vivre chacun à la limite, on the edge. Cela provoque une envie supplémentaire qui permet de voir plus loin, évitant ainsi la sensation d'être «blasé», très ressentie parmi les jeunes élèves des programmes similaires en Europe. $^{20}$

Mais il n'y a pas que la défamiliarisation du nouveau qui évite la démotivation due à la monotonie. C'est également tout un travail de médiation qui se fait entre ce qui est acquis, relevant de la confiance aux «attachements» (Hennion, 2013) ${ }^{21}$, et ce qui est nouveau, à atteindre, relevant de la «défamiliarisation». Chronologiquement il est difficile de savoir ce qui advient en premier. Est-ce que l'attachement à quelque chose donne confiance pour aller plus loin? Faut-il, dès le début, vaincre une première défamiliarisation pour aboutir à un attachement? C'est là que se mêlent des aspects très divers liés aux attachements chez le jeune musicien d'un orchestre. Voici

\footnotetext{
${ }^{17}$ Terme employé par Shklovsky dans l'opposition entre la prose et la poésie (Shklovsky, 2008).

${ }^{18}$ Cependant, cela devient moins vrai après des décennies de travail dans un même territoire.

19 Terme choisi par le traducteur Régis Gayraud (Shklovsky, 2008).

${ }^{20}$ Observations sur le terrain auprès des orchestres Démos (Paris) en 2010 et Geração (Lisbonne) en 2011 et 2016. C'est aussi un ressenti verbalisé par les professeurs vénézuéliens qui enseignent en Europe également.

21 «Attachement», lié à la théorie de l'attachement en Psychologie. Employé ici selon le travail réalisé par Antoine Hennion autour de ce concept adapté à la sociologie: les faits implicites et explicites qui lient un collectif, ou qui lient une personne à ses «passions» (Hennion, 2013).
} 
trois situations recueillies sur le terrain, parmi tant d'autres : (1) le musicien intègre un orchestre parce-que ses parents (auxquels il est attaché) lui ont conseillé de rejoindre cette initiative, il le fait pour satisfaire leur volonté, pour faire plaisir à ceux à qui il est lié; (2) le musicien intègre un orchestre parce-que son meilleur ami, voisin du barrio, y est déjà; (3) le musicien intègre un orchestre parce-que cela "fait bien" de se balader dans le quartier avec un violon sous le bras.

Pour le jeune élève, ces attachements (Hennion, 2004) donnent l'élan nécessaire pour se confronter à la difficulté de la défamiliarisation provoquée par la musique symphonique et ses partitions, par les classes de cinquante élèves, l'autorité du maestro, les longues heures de cours (jusqu'à cinq heures par jour). Les attachements sont essentiels, notamment parce qu'ils dépendent de processus extrêmement longs. C'est un point clé dans la perspective de Shklovsky: «Un objet d'art est créé artistiquement pour que sa perception soit retardée, et le meilleur effet possible est celui produit au cours de la lenteur de la perception» (2008).

Il existe toute une médiation entre la défamiliarisation et l'attachement. L'union constante des deux faits permet de travailler dans la ligne de "l'étrangement familier". Non pas au sens freudien mais au sens de l'élan, du momentum que crée ce mélange entre la sécurité de l'attachement et le risque de l'étrangisation. Sur le terrain les professeurs du programme El Sistema jouent beaucoup sur cet équilibre au niveau de l'expérience même: "Le propos de l'art est de conférer la sensation des choses au moment où elles sont perçues physiquement et non pas au moment où elles sont connues» (Shklovsky, 2008)

\section{Discussion et conclusion}

L'attachement et la défamiliarisation font tous deux partie intégrante de l'expérience esthétique, autant artistique que sociale. Ces deux conceptualisations s'échangent le rôle de ce que Dewey nomme «impulsion» (2010: 87), proche du punctum théorisé par Barthes (1980), c'est-à-dire quelque chose de subtil et de personnel, essentiel pour que le jeune élève d'El Sistema puisse maintenir son élan d'apprentissage.

Partons d'un exemple pratique de ce va-et-vient permanent entre l'attachement qui familiarise et l'étrangéité qui défamiliarise: au Venezuela les professeurs des orchestres de jeunes dans les écoles des barrios sont souvent eux-mêmes issus des quartiers; ils sont donc connus et reconnus par les jeunes élèves, ce qui crée un premier attachement (i.e. partage d'un territoire, connaissance entre familles, jeux de pelota de goma en fin de journée). Cet attachement est renforcé lorsque le professeur, venant des mêmes conditions socioculturelles, a réussi à atteindre un haut niveau musical et joue pour l'un des grands orchestres en tournée à travers le monde. ${ }^{22}$ Pour l'élève, cet attachement conforte et renforce le désir de réussir, il sert alors de tremplin pour vaincre la défamiliarisation d'une partition orchestrale ou de la méthode Suzuki. ${ }^{23}$

Un autre point qui me semble important dans l'analyse proposée ici, c'est le fait que la défamiliarisation puisse être elle-même un élément d'attachement. Je m'en suis souvent aperçu

\footnotetext{
${ }^{22}$ Maestro Gustavo Dudamel, jeune prodige vénézuélien reste un modèle à suivre pour toute la jeunesse du núcleo Santa Rosa de Agua, mais il y a aussi des cas plus proches comme par exemple un violoncelliste du même quartier périphérique qui a été admis au Conservatoire de Paris. La proximité du modèle à suivre est fondamentale, notamment parce-que c'est une preuve matérielle de la possibilité de changement.

${ }^{23}$ Shinichi Suzuki (1898-1998), violoniste et auteur d'une méthode d'apprentissage de la musique. Sa pédagogie est employée dans beaucoup d'écoles et conservatoires.
} 
dans les observations ethnographiques sur le terrain lorsque, par exemple, les jeunes élèves apportent de nouvelles partitions au professeur (trouvées sur Internet) et que celles-ci sont d'un niveau bien supérieur au leur : les professeurs ne coupent pas cet élan naïf, au contraire, ils poussent les élèves à faire face au «choc» de la complexité. C'est la même chose au niveau de la technique physique pour jouer d'un instrument lorsque les jeunes musiciens parlent au professeur

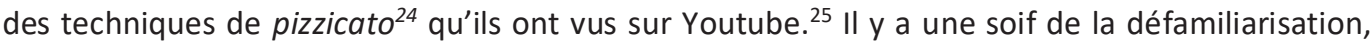
compétitive même, puisque chacun cherche à être le premier à savoir jouer une suite de Bach au violoncelle ou à contrôler la respiration continue avec sa clarinette.

Pour Shklovsky "L'art comme procédé récuse la dichotomie de la forme et du fond» (2008). C'est vérifiable dans les échanges permanents entre attachement et défamiliarisation analysés plus haut. Le processus, tout en étant formel, d'ordre structurel et physique même, véhicule lui aussi un fond artistique - il y a du "poétique" tout le long du processus. C'est à ce niveau qu'il est extrêmement complexe de vouloir recréer le programme El Sistema dans d'autres contextes tels que les banlieues nord de Paris (e.g. Programme Demos - Amico et al., 2018; Virginie D’Eau, 2012) ou la périphérie de Lisbonne (e.g. Programme Orquestra Geração - Elvas, 2010; Mota \& Teixeira Lopes, 2017).

El Sistema se sert de l'orchestre symphonique comme structure formelle où tout un ensemble de médiations à caractère pédagogique (aux niveaux social et esthétique) peut être développé. Cette structure a en effet des piliers porteurs (l'enseignement en collectif, chacun est à la fois élève et professeur, le travail doit être quotidien et intensif, etc.), mais le fond «poétique» est propre à chaque contexte. Ainsi il est impossible de reproduire l'ordre structurel d'El Sistema, d'abord parce que cet ordre n'est pas défini et ensuite parce que cela serait en rester à un niveau "prosaïque», oubliant la vitalité du rythme «poétique» propre à chaque territoire, comme le soutien Shklovsky:

Il y a en effet de l'ordre dans l'art, mais pourtant aucune colonne de temple grec ne s'élève dans l’ordre ; le rythme poétique est lui aussi désordonné. Les tentatives pour systématiser les irrégularités ont été faites, et ces tentatives font partie des problèmes actuels en théorie du rythme. Il est clair que la systématisation ne va pas marcher, car en ce qui concerne la réalité le problème n'est pas celui de la complexification du rythme mais de créer du désordre dans le rythme - un désordre qui ne peut pas être prédit. (Shklovsky, 2008)

Cette proposition de Shklovsky d'ouverture à la poétique du désordre est difficilement acceptable par l'académie en général et les sciences sociales en particulier car notre travail est de tenter de démystifier, d'expliquer en "décortiquant" les faits sociaux. C'est toute la difficulté de notre mission en tant que chercheurs en sociologie - expliquer sans rompre la magie, concrétiser sans figer. Pour conclure en intégrant l'ensemble des acteurs qui forment un núcleo au Venezuela ou une école de musique à travers le monde, je dirais que les questions énoncées au cours de cet article pourraient être rassemblées et alignées de la façon suivante : Quels sont les attachements qui comptent? Y a-t-il une hiérarchie entre eux? De quelle façon sont-ils mêlés? Est-ce que les attachements contribuent à embrasser l'exercice de la défamiliarisation? A partir de l'observation ethnographique, des entretiens semi-structurés et des focus-groups, il est possible d'élargir nos

\footnotetext{
${ }^{24}$ Technique employée sur les instruments à cordes. Cela consiste à pincer les cordes avec sa main droite au lieu de se servir d'un archet.

${ }^{25}$ Les barrios le plus pauvres ont aussi les réseaux Internet et les jeunes musiciens en profitent pour consulter des cours en ligne.
} 
connaissances sur les complexités des interactions sociales entre tous les acteurs d'un núcleo. Il est aussi possible d'analyser avec un détail pragmatiste les possibles atouts de l'outil pédagogique que peut être l'orchestre symphonique. La forme et le fond se mêlent dans les mécanismes artistiques. Tous deux prennent constamment la relève pour garantir le momentum de l'apprentissage chez les jeunes musiciens d'El Sistema, dépendant d'un équilibre entre la sécurité des attachements et le choc de la défamiliarisation.

\section{RÉFÉRENCES BIBLIOGRAPHIQUES}

Agrech, Vincent (2018). Un orchestre pour sauver le monde? Paris: Stock.

Amico, Marta; Clouet, Claire; le Calvé, Maxime; Lewandowski, Julie, Lisack, Lucille \& Oleksiak, Julie (2018). Des enfants musiciens comme projet de société ? Le projet Démos de la Philharmonie de Paris. Cahiers d'ethnomusicologie, 31, pp. 119-136.

Arvelo, Alberto (2006). Tocar y luchar. Venezuela: Studios: Explorart Films.

Baker, Geoffrey (2014). El Sistema : orchestrating Venezuela's youth. New York: Oxford University Press.

Barthes, Roland (1980). La chambre claire. Note sur la photographie. In Cahiers du cinéma. Paris: Gallimard.

Becker, Howard S. (1974). Art As Collective Action. American Sociological Review, 39(6), pp. 767-776.

Benjamin, Walter (2000). Sur quelques thèmes baudelairiens. In CEuvres III (pp. 329-390). Paris: Gallimard.

Caune, Jean (2006). La démocratisation culturelle, une médiation à bout de souffle. Grenoble: Presses Universitaires de Grenoble.

Cefaï, Daniel (2007). Pourquoi se mobilise-t-on ? Les théories de l'action collective. Paris: La Découverte.

Creech, Andrea (2016). El Sistema and Sistema-Inspired Programmes: A Literature Review of research, evaluation, and critical debate. San Diego: Sistema Global.

Das, Bijay Kumar (2010). Twentieth Century Literary criticism (6th ed.). New Delhi: Atlantic Publishers \& Distributors Pvt Ltd.

Dessons, Gérard (2004). L'art et la manière : art, littérature, langage. Paris: Honoré Champion.

Dewey, John (2010). L'art comme expérience. In Collection Folio Essais (Folio). Paris: Gallimard.

Elvas, Isabel (2010). A Orquestra Geração na Escola Básica 2,3 Miguel Torga na Amadora. Revista Migrações Número Temático Música e Migração, 7, pp. 293-294.

Eyries, Alexandre (2014). Communication politique et culture: enjeux paradoxaux de la médiation culturelle impulsée par André Malraux. Quaderni, (83), pp. 83-90.

Govias, Jonathan Andrew (2011). The five fundamentals of El Sistema. Canadian Music Educator, pp. 21-23. Retrieved from https://jonathangovias.files.wordpress.com/2012/09/govias-five-fundamentals-of-elsistema.pdf

Hennion, Antoine (1988). Comment la musique vient aux enfants: une anthropologie de l'enseignement musical. Paris: Anthropos-Economica.

Hennion, Antoine (2004). Une sociologie des attachements. D'une sociologie de la culture à une pragmatique de l'amateur. Societes, 2004(85 3), pp. 9-24.

Hennion, Antoine (2007). La passion musicale : une sociologie de la médiation. Paris: Métailié.

Hennion, Antoine (2013). D'une sociologie de la médiation à une pragmatique des attachements. Sociologies [En ligne], Théories et recherches, mis en ligne le 25 juin 2013, consulté le 03 septembre 2020. URL : http://journals.openedition.org/sociologies/4353

Hennion, Antoine (2015). Enquêter sur nos attachements. Comment hériter de William James ? SociologieS [En ligne], Dossiers, Pragmatisme et sciences sociales : explorations, enquêtes, expérimentations, mis en ligne le 23 février 2015, consulté le 03 septembre 2020. URL : http://journals.openedition.org/sociologies/4953

Lafortune, Jean-Marie (2012). La médiation culturelle : le sens des mots et l'essence des pratiques. Montréal: Presses de l'Université du Québec.

Mauss, Marcel (1950). Les techniques du corps (1936). In Sociologie et anthropologie (pp. 365-386). Paris: PUF.

Mota, Graça \& Teixeira Lopes, João (2017). Crescer a tocar na Orquestra Geração. Porto: Verso da História.

Rojas, Eduardo G. (2010). Estilo gerencial de la Fundación del Estado para el Sistema Nacional de Orquestas Juveniles e Infantiles de Venezuela. Caso en estudio: estado lara. Barquisimeto, Venezuela: Universidad Centroccidental Lisandro Alvarado.

Sarrouy, Alix Didier (2011). Médiation socioculturelle - comprendre et définir ses fonctions en partant d'un cas concret : l'adaptation du model d'éducation musicale El Sistema à de nouveaux contextes. Mémoire pour Master 1- Paris: Université Sorbonne Nouvelle - Paris III.

Sarrouy, Alix Didier (2016). Atores da educação musical: etnografia comparativa entre três núcleos que se inspiram no programa EI Sistema na Venezuela, no Brasil e em Portugal. Braga: Universidade do Minho.

Sarrouy, Alix Didier (2017a). Acteurs de l'éducation musicale : ethnographie comparative entre trois núcleos qui s'inspirent du programme El Sistema au Venezuela, au Brésil et au Portugal. Paris: Université Sorbonne Nouvelle - Paris III. 
Sarrouy, Alix Didier (2017b). Adapter pour mieux adopter - Ethnographie dans des núcleos d'éducation musicale au Venezuela et au Portugal. Sociologia OnLine, (15), pp. 15-30. https://doi.org/00.0000/SON2017XXXXXXX

Sarrouy, Alix Didier (2018). Actores de la continuidad educativa en barrios de Venezuela: madres del núcleo Santa Rosa de Agua. Comparative Cultural Studies, (5), pp. 43-54.

Scripp, Lawrence (2015). The need to testify: A Venezuelan musician's critique of El Sistema and his call for reform. Retrieved from www.researchgate.net/publication/285598399

Shklovsky, Viktor (1988). Art as technique. Modern Criticism and Theory: A Reader, 11, pp. 16-30.

Shklovsky, Viktor (2008). L'Art comme procédé. (Petite Col). Paris: ALLIA.

Shusterman, Richard (1991). L'art a l'état vif, la pensée pragmatiste et l'esthétique populaire. Paris: Les Éditions de Minuit.

Silberman, Lauren R. (2013). Globalizing El Sistema! Exploring the growth and development of El Sistema inspired programs around the world. Eugene: University of Oregon.

Smaczny, P., \& Stodmeier, M. (2009). El Sistema: music to change life. France: Producteurs: EuroArts Music International. Coproduit par ARTE France, NHK.

Tunstall, T., \& Booth, E. (2016). Playing for their lives. New York: W. W. Norton \& Company.

Uy, Michae (2012). Venezuela's national music education program El Sistema: its interactions with society and its participants' engagement in Praxis. Music \& Arts in Action, (4(1)), pp. 5-21.

Virginie D’Eau. (2012). Rapport Final d'Evaluation du Projet DEMOS. Retrieved from http://www.observatoireculture.net/fichiers/files/etude_integrale_telecharger_2.pdf

Alix Sarrouy. Doutor em Sociologia da Arte e da Cultura. Investigador em Ciências Sociais, Músico \& Performer. Licenciado em Mediação Cultural (Paris III), mestre em Políticas Culturais (Paris VII), com duplo doutoramento em Sociologia da Arte e da Cultura (Paris III \& Universidade do Minho). NOVA FCSH, Colégio Almada Negreiros, Campus de Campolide, 3. piso - Sala 333, 1070-312 Lisboa, Portugal. E-mail: alixsarrouy@fcsh.unl.pt. ORCID: 0000-0003-0574-7819.

Financement: Cette recherche a été possible grâce aux financements suivants: Marie Skłodowska-Curie actions (FP7-SP3-PEOPLE), Multilevel Governance of Cultural Diversity in a Comparative Perspective: EU-Latin America; Programme d'Actions Universitaires Intégrées Luso-Françaises; Aide à la Mobilité Internationale des Doctorants, Conseil Régional D’lle-de-France.

Remerciements: Je remercie les réviseurs anonymes pour leurs conseils avisés.

Receção: 04-02-2020

Aprovação: 12-06-2020

Citação:

Sarrouy, Alix (2020). El Sistema et l'attachement à la défamiliarisation: enseignement et apprentissage de la musique symphonique à Santa Rosa de Agua, Venezuela. Todas as Artes. Revista Luso-brasileira de Artes e Cultura, 3(1), pp. 33-47. ISSN 2184-3805. DOI: 10.21747/21843805/ta3n1a3 